\title{
Bounds for shear viscosity in Nabarro-Herring-Coble creep
}

\author{
Laurence Brassart $^{\mathrm{a}, *}$, Francis Delannay $^{\mathrm{b}}$ \\ ${ }^{a}$ Department of Materials Science and Engineering, \\ Monash University, Clayton, VIC 3800, Australia \\ ${ }^{b}$ Institute of Mechanics, Materials and Civil Engineering (iMMC/IMAP), \\ Université catholique de Louvain, B-1348 Louvain-la-Neuve, Belgium
}

\begin{abstract}
At high homologous temperature, plastic flow of a polycrystalline material can be mediated by self-diffusion and grain boundary sliding, rather than by dislocation glide. The effective viscosity of the polycrystal depends on the underlying mechanisms as well as on the microstructure. Simple expressions relating the shear viscosity to lattice and grain boundary diffusion coefficients were proposed in pioneering contributions by Nabarro and Herring [Herring, C., 1950. J. Appl. Phys. 21, 437-445] and Coble [Coble, R., 1963. J. Appl. Phys. 34, 1679-1682]. While these models remain widely used today to deduce the dominant mechanism based on the observed dependence of viscosity on grain size, a number of questions remain open. The present work revisits Nabarro-HerringCoble creep using a micromechanical approach and variational principles. We focus on a random polycrystal of equiaxed grains deforming by coupled lattice and grain boundary diffusion and grain boundary sliding. We show that the classical results of Herring and Coble correspond to upper bounds on the shear viscosity, and obtain complementary lower bounds. Our results shed new light on these classical results and question the validity of the common interpretation of the dependence of viscosity on grain size.

Keywords: Diffusional creep, Grain boundary sliding, Effective behaviour, Variational principles
\end{abstract}

\section{Introduction}

Nabarro (Nabarro, 1948) and Herring (Herring, 1950) were the first to propose that, under low stress, creep of a polycrystalline solid can proceed without dislocation activity by diffusional flow from grain boundaries under normal compression toward grain boundaries under normal tension. Owing to the linear dependence of the diffusion potential on the stress normal to a grain boundary (Herring, 1950; Green, 1970; Herring, 1971; Balluffi et al., 2005), such a deformation mechanism should bring about a linearly viscous macroscopic behaviour. According to Herring's model (Herring, 1950), if diffusion occurs only through the bulk of the lattice, the macroscopic viscosity is proportional to the square of grain size. Noticing that diffusion at low homologous temperature may be restricted to a thin layer close to grain boundaries, Coble (Coble, 1963) complemented Herring's model by showing that viscosity should then vary as the third power of grain size. The Nabarro-Herring-Coble (NHC) creep model has been very widely adopted in the materials community and the pioneering papers of Nabarro, Herring and Coble remain today very

\footnotetext{
*Corresponding author. Email: laurence.brassart@monash.edu
} 
commonly cited in the literature, in particular in relation to the grain-size dependence of creep rate and sintering rate.

Based on these papers, many authors have worked out models for establishing the link between the macroscopic creep rate and the mechanisms underlying NHC creep (Gibbs, 1966; Green, 1970; Raj and Ashby, 1971; Beere, 1976, 1977, 1978; Spingarn and Nix, 1978; Greenwood, 1985, 1992; Pan and Cocks, 1993; Burton, 1994; Cocks, 1996; Mori et al., 1997; Keblinski et al., 1998; Mori et al., 1998a; Onaka et al., 1998; Mori et al., 1998b; Onaka et al., 2001; Kim et al., 2003, 2004; Wei et al., 2008; Wang et al., 2011; Hötzer et al., 2019). The objective of the present paper is to shed a new light on the dependence of NHC creep rate on material parameters via the use of variational principles, which define upper and lower bounds for the macroscopic viscosity, $G$, of a polycrystal (Needleman and Rice, 1980; Cocks, 1994). Although reference to such bounds has already been made in connection with modelling of diffusional deformation (Ashby et al., 1978; McMeeking and Kuhn, 1992; Cocks, 1996), what these bounds imply for NHC creep has not been thoroughly analysed yet. It will be shown that the NHC model actually corresponds to an upper bound for $G$, and that complementary lower bounds can also be obtained. The results will question in particular the validity of the classical interpretation of the dependence of viscosity on grain size.

The basics of the NHC creep mechanism are that a velocity difference between adjacent grains in direction normal to grain boundary can arise via the emission or absorption of vacancies at grain boundaries, where all sources and sinks of vacancies are supposed to be localised. At the microscopic scale, the mechanism can be apprehended in terms of climb of grain boundary dislocations (GBDs) (Gleiter et al., 1968) having a Burgers vector perpendicular to the boundary. The rate-governing parameters of dislocation climb are the vacancy diffusivities, in the bulk of the lattice and in a layer of thickness $\delta$ along the grain boundary. Ashby and co-workers have discussed in terms of GBDs the imperfect character of the grain boundary as source and sink of vacancies, which brings about departure from purely Newtonian viscous behaviour (Ashby, 1969, 1972; Arzt et al., 1983).

However, Lifshitz (Lifshitz, 1963) was the first to emphasize the fact that it is not possible to account for the component of velocity difference parallel to grain boundary if emission/absorption of vacancies is not accompanied by sliding along grain boundaries. He proposed to represent the resistance to grain boundary sliding by a linear law:

$$
\sigma_{t}=\eta \Delta v_{t}
$$

where $\sigma_{t}$ is the shear stress tangent to grain boundary, $\Delta v_{t}$ is the tangent component of the grain velocity difference, and $\eta$ is a friction coefficient. It follows that macroscopic viscosity involves, in addition to the dissipation considered by Herring and Coble, a contribution due to resistance to grain boundary sliding. If $\eta$ is large, dissipation could become dominated by the latter contribution and viscosity would then tend to vary linearly with grain size.

At the microscopic scale, the mechanism of grain-boundary sliding can be apprehended in terms of glide of GBDs having Burgers vector parallel to the boundary (Ashby, 1972; Crossman and Ashby, 1975; Bilde-Sørensen and Smith, 1994). The friction coefficient $\eta$ should then be linked to the critical resolved shear stress for GBD glide, which is largely unknown and not accessible experimentally. In early models, authors circumvented this difficulty by advocating that dissipation due to grain boundary friction should be negligible with respect to dissipation due to diffusion because GBD glide amounts to the displacement of a collection of atoms at the scale of lattice parameter whereas GBD climb 
requires vacancy diffusion at grain scale (Gibbs, 1968; Burton, 2002). However, as noticed by Beere (Beere, 1977, 1978), periodic arrays of parallelepipeds deforming according to the NHC mechanism would collapse like a pack of cards if boundary friction stresses were null. Analyses taking full account of the coupling of diffusion and frictional sliding at grain boundaries were proposed by Mori and co-workers (Mori et al., 1998a,b; Onaka et al., 1998, 2001) and by Kim and co-workers (Kim et al., 2004, 2005a,b, 2009).

Beside the role of boundary friction forces, question marks also aroused from experimental observations revealing that the magnitude of (i) grain boundary sliding measured by line tracing methods (Gifkins and Langdon, 1970) and (ii) grain elongation measured after superplastic forming are, respectively, larger and smaller than what should correspond to the NHC mechanism. This issue led Cannon (Cannon, 1972) to introduce the distinction between two types of grain boundary sliding: "Lifshitz sliding", which is the sliding required for the respect of compatibility conditions between adjacent grains during the NHC deformation mode, and "Rachinger sliding", which brings about a different deformation mode involving grain rearrangement by relative motion of grains that remain equiaxed (Rachinger, 1952-1953). Like most former models in literature, the present paper considers only Lifshitz sliding. Extension of the approach to Rachinger sliding will be the subject of another paper.

After introducing in Section 2 the governing equations describing local and effective flow behaviours of polycrystals, we present in Section 3 the basics of the variational formulation of the problem, which brings to the concepts of kinematic/upper bounds and statical/lower bounds for the shear viscosity. In Section 4, these concepts are applied to the modelling of deformation of a randomly isotropic polycrystal in conditions of coexistence of lattice diffusion and grain boundary diffusion. Like in Herring's model, the representative unit cell is taken to be a spherical grain. Variational principles yield expressions for the dependence of upper and lower bounds of $G$ on grain size, lattice and grain boundary diffusion coefficients $D_{l}$ and $D_{b}$, and friction coefficient $\eta$. Whereas the upper bound expression largely agrees with former models in the literature, the expression for the corresponding lower bound has not been reported previously. The results are discussed in Section 5, which focuses in particular on the dependence of the grain size sensitivity exponent on $\eta$.

\section{Governing equations}

\subsection{Local behaviour}

We consider a dense aggregate of rigid grains deforming by a combination of diffusional creep and grain boundary sliding (Fig. 1a). Diffusion in the bulk of the grains is mediated by a vacancy-exchange mechanism, and we neglect the creation and annihilation of vacancies within the bulk. The driving force for atomic diffusion is the gradient of the difference between the chemical potential of the atoms, $\mu_{A}$ and that of the vacancies, $\mu_{V}$. Introducing the diffusion potential $\mu=\mu_{A}-\mu_{V}$, we express the lattice (volumetric) diffusion flux (in units of length/time) as (Herring, 1950):

$$
\boldsymbol{j}_{l}=-\frac{D_{l}}{k T} \nabla \mu,
$$

where $k$ is Boltzmann's constant and $T$ is the absolute temperature. The lattice diffusion coefficient $D_{l}$ (in units of length ${ }^{2} /$ time) is related to the vacancy diffusion coefficient $D_{V}$ by $D_{l}=D_{V} X_{V}$, where $X_{V}$ is the atom fraction of vacancies (Balluffi et al., 2005). We 
assume that variations of $X_{V}$ throughout the crystal are small, and treat the diffusion coefficient $D_{l}$ as a constant. Assuming that diffusion is in quasi steady-state, the equation of conservation of atoms writes:

$$
\nabla \cdot \boldsymbol{j}_{l}=0 .
$$

It follows from Eqs (2) and (3) that $\nabla^{2} \mu=0$ in the bulk of a grain. The diffusion potential on the boundary is related to the normal stress $\sigma_{n}$ by (Herring, 1950, 1971):

$$
\mu=\mu_{A}^{\circ}-\Omega \sigma_{n}-\mu_{V}=\mu_{A}^{\circ}-\Omega \sigma_{n},
$$

where $\mu_{A}^{\circ}$ is a reference chemical potential (usually taken as the free energy per atom in an infinitely-extended, vacancy-free crystal) and $\Omega$ is an average volume per atom. In the rest of the paper, we will set $\mu_{A}^{\circ}=0$, without loss of generality. Expression (4) describes the reduction in potential energy of the system due to the insertion of an atom below the boundary under traction. The second equality in Eq. (4) follows from the assumption that grain boundaries act as ideal sources and sinks for vacancies, so that the vacancies are maintained at their equilibrium concentration near the interface and $\mu_{V}=0$ (Balluffi et al., 2005).

The grain boundary is considered to consist of a thin layer of thickness $\delta$ in which the diffusion coefficient $D_{b}$ (in units of length ${ }^{2} /$ time), is different from the lattice diffusion coefficient $D_{l}$. The volumetric diffusion flux inside the grain boundary (in units of length/time) is given by:

$$
\boldsymbol{j}_{b}=-\frac{\delta D_{b}}{k T} \nabla_{s} \mu
$$

where $\nabla_{s}$ denotes the gradient operator on the grain boundary surface and $\mu$ is given by Eq. (4). Conservation of matter at grain boundary junctions requires that:

$$
\sum \boldsymbol{j}_{b} \cdot \boldsymbol{m}=0
$$

where the summation extends over the grain boundaries meeting at a junction and $\boldsymbol{m}$ is the outward normal to the arc of intersection within the grain boundary plane of each participating grain facet.

The difference of velocity, $\Delta v_{n}$ between adjacent grains in the direction normal to the grain boundary is supposed to have only two possible origins: the normal component of $\boldsymbol{j}_{l}$ on the surface of each grain, and the divergence of $\boldsymbol{j}_{b}$ in the grain boundary surface:

$$
\Delta v_{n}=-\left(\boldsymbol{j}_{l}^{(+)}-\boldsymbol{j}_{l}^{(-)}\right) \cdot \boldsymbol{n}-\boldsymbol{\nabla}_{s} \cdot \boldsymbol{j}_{b},
$$

where the superscripts $(-)$ and $(+)$ refer to the two adjacent grains and $\boldsymbol{n}$ is the outward normal to grain $(-)$ pointing towards grain $(+)$, see Fig. 1b. The tangential difference of velocity, $\Delta v_{t}$ between adjacent grains is supposed to be linearly related to the tangential stress on the grain boundary surface:

$$
\Delta v_{t}=\frac{1}{\eta} \sigma_{t}
$$

where $\sigma_{t}$ is the shear stress on the surface and $\eta$ is a friction coefficient characterizing the sliding mechanism operating at microscopic scale.

The field of stress must satisfy mechanical equilibrium in grain interior, $\boldsymbol{\nabla} \cdot \boldsymbol{\sigma}=\mathbf{0}$, and tractions must equilibrate at grain boundaries: $\left(\boldsymbol{\sigma}^{(-)}-\boldsymbol{\sigma}^{(+)}\right) \cdot \boldsymbol{n}=\mathbf{0}$. We also assume that the internal grain-boundary tensions are at equilibrium. Mechanical boundary conditions can be written in terms of prescribed velocity or prescribed traction components on the external surface of the aggregate. Chemical boundary conditions can be specified in terms of prescribed net flux or prescribed diffusion potential on the external surface. 


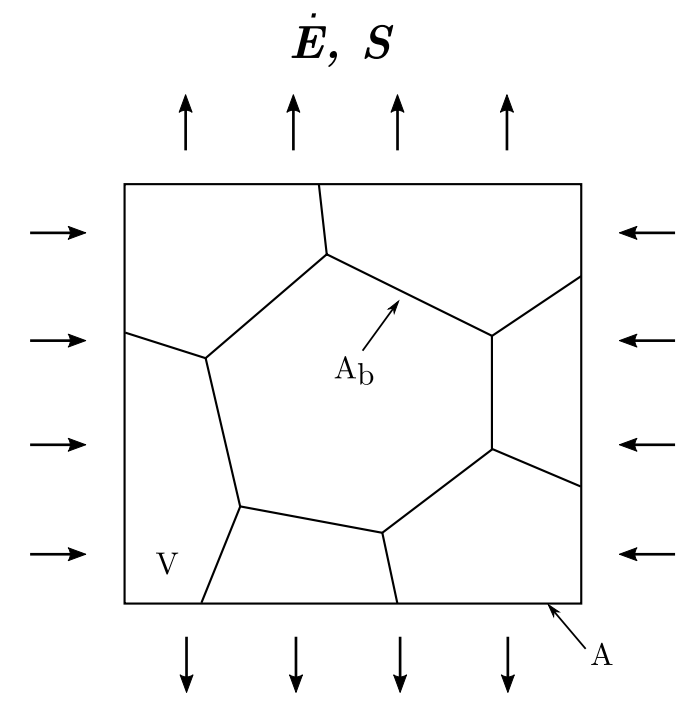

(a)

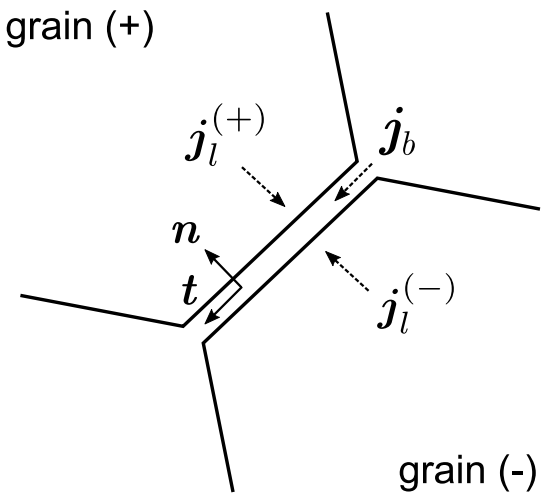

(b)

Figure 1: (a) An aggregate of grains subject to a macroscopic strain rate $\dot{\boldsymbol{E}}$ or a macroscopic stress $\boldsymbol{S}$ via prescribed velocity or prescribed traction boundary conditions applied to the external surface $A$. The aggregate contains grains with total volume $V$ meeting at grain boundaries with total surface $A_{b}$. (b) Deformation of the aggregate is mediated by surface diffusion along the grain boundaries, lattice diffusion within the grains and sliding along grain boundaries.

\subsection{Effective behaviour}

The effective behaviour of the aggregate is described by the relationship between the average (macroscopic) strain rate $\dot{\boldsymbol{E}}$ and the average stress $\boldsymbol{S}$ in a Representative Volume Element (RVE) of the aggregate. We focus on steady-state creep, i.e. we assume that all transient contributions to stress and diffusion fields during initial elastic loading of the polycrystal have relaxed. Relaxation kinetics towards steady-state was previously investigated in 2D by Mori et al. (1998a) and Onaka et al. (1998). Accounting for the velocity discontinuity at the grain boundaries, the macroscopic strain rate is defined as:

$$
\begin{aligned}
\dot{E}_{i j} & =\frac{1}{2 V} \int_{A}\left(v_{i} n_{j}+v_{j} n_{i}\right) d A \\
& =\frac{1}{2 V} \int_{A_{b}}\left(\Delta v_{i} n_{j}+\Delta v_{j} n_{i}\right) d A
\end{aligned}
$$

where $V$ is the total volume of the aggregate, $A$ denotes the external surface of the aggregate and $A_{b}$ denotes the grain boundary area, see Fig. 1a. In the case of deformable grains and when the velocity field is continuous, the first equality is equivalent to the usual definition of macroscopic strain rate as the volume average of the microscopic strain rate. The second equality is obtained using the divergence theorem and accounting for the rigidity of the grains.

The macroscopic stress is defined as:

$$
\begin{aligned}
S_{i j} & =\frac{1}{2 V} \int_{A}\left(T_{i} x_{j}+T_{j} x_{i}\right) d A \\
& =\frac{1}{V} \int_{V} \sigma_{i j} d V
\end{aligned}
$$


where $x_{i}$ is the position vector and $T_{i}=\sigma_{i j} n_{j}$ the traction vector on the external surface of the aggregate. The second equality follows from the equilibrium of tractions across grain boundaries and the condition of mechanical equilibrium in the grain interior.

In a classical micromechanics approach, the effective behaviour of the aggregate is obtained as follows. In the case where the macroscopic strain rate $\dot{\boldsymbol{E}}$ is prescribed, velocity boundary conditions are chosen such that equality (9) is satisfied. After solving the boundary-value problem, the macroscopic stress $\boldsymbol{S}$ is calculated using Eq. (12). Alternatively, in the case where the macroscopic stress $\boldsymbol{S}$ is prescribed, traction boundary conditions are chosen such that equality (11) is satisfied. After solving the boundary-value problem, the macroscopic strain rate $\dot{\boldsymbol{E}}$ is calculated using Eq. (10). We assume that Hill's condition holds (Hill, 1967):

$$
V \boldsymbol{S}: \dot{\boldsymbol{E}}=\int_{A} \boldsymbol{T} \cdot \boldsymbol{v} d S
$$

which states the equality of the macroscopic and average microscopic powers expended in the RVE. This condition is satisfied by affine velocity boundary conditions for a prescribed strain rate, $\boldsymbol{v}=\dot{\boldsymbol{E}} \cdot \boldsymbol{x}$ on $A$, and by uniform traction boundary conditions for a prescribed macroscopic stress, $\boldsymbol{T}=\boldsymbol{S} \cdot \boldsymbol{n}$ on $A$.

We verify that the effective behaviour resulting from the local equations summarised in Section 2 is incompressible. According to Eq. (10), the volumetric strain rate is related to the normal velocity discontinuity at grain boundaries by:

$$
V \dot{E}_{k k}=\int_{A_{b}} \Delta v_{n} d A
$$

Using the continuity equation (7) and the divergence theorem, we obtain:

$$
\begin{aligned}
V \dot{E}_{k k} & =\int_{\Gamma_{b}}-\sum\left(\boldsymbol{j}_{b} \cdot \boldsymbol{m}\right) d L+\int_{V} \boldsymbol{\nabla} \cdot \boldsymbol{j}_{l} d V \\
& =0
\end{aligned}
$$

In writing Eq. (15), we have implicitly assumed zero-net lattice and boundary flux boundary conditions. The first term on the right-hand side vanishes according to Eq. (6) and the second term vanishes according to Eq. (3). Incompressibility implies that, for prescribed strain rate, the macroscopic stress can only be calculated up to an undetermined hydrostatic stress. If the aggregate is isotropic, the effective behaviour is fully characterised by the macroscopic shear viscosity $G$, defined as:

$$
G=\frac{S_{i j}^{\prime}}{2 \dot{E}_{i j}^{\prime}}=\frac{S_{i j}^{\prime}}{2 \dot{E}_{i j}},
$$

where the notation $(\cdot)^{\prime}$ refers to the deviatoric part of a second-order tensor. The macroscopic bulk viscosity $K$ is infinite.

\section{Variational principles}

The governing equations presented in the previous section can be recast in variational form. The kinematic variational principle given below was first derived by Needleman and Rice (1980) for coupled grain-boundary diffusion and dislocation-mediated plasticity. Several variations were subsequently proposed to also describe grain-boundary sliding (McMeeking and Kuhn, 1992), interface reaction (Cocks, 1994) and lattice diffusion 
(Cocks, 1996). Complementary statical variational principles were derived by Cocks (1994, 1996). The variational principles form the basis of Galerkin-type finite-element procedures for simulating the evolution of material surfaces at microscopic scale, see for example (Pan and Cocks, 1993; Suo, 1997; Cocks et al., 1998) or more recently (Wei et al., 2008). Here, we use variational principles in order to obtain bounds on the effective shear viscosity.

\subsection{Kinematic variational principle}

Consider an aggregate subject to prescribed velocity boundary conditions on the portion $A_{V}$ of its external surface, and to prescribed traction boundary conditions on the portion $A_{T}$ of its external surface, such that $A_{V} \cup A_{T}=A$ and $A_{V} \cap A_{T}=\emptyset$. The kinematic variational principle states that, among all the kinematically admissible velocity fields and diffusion flux fields, the actual fields minimise the following functional:

$$
I=\Psi-\frac{1}{V} \int_{A_{T}} \overline{\boldsymbol{T}} \cdot \boldsymbol{v} d A,
$$

where $\Psi$ is a dissipation potential given by:

$$
\Psi=\frac{1}{V}\left[\int_{V} \frac{1}{2} \frac{k T}{\Omega D_{l}} \boldsymbol{j}_{l} \cdot \boldsymbol{j}_{l} d V+\int_{A_{b}}\left(\frac{1}{2} \frac{k T}{\Omega \delta D_{b}} \boldsymbol{j}_{b} \cdot \boldsymbol{j}_{b}+\frac{1}{2} \eta\left(\Delta v_{t}\right)^{2}\right) d A\right] .
$$

The second term of Eq. (18) represents the potential energy of the applied tractions, with $\overline{\boldsymbol{T}}$ the prescribed traction vector on $A_{T}$. An admissible velocity field is such that its gradient vanishes in the grain interior (rigid grains), and such that the normal velocity jump across grain boundaries is related to the diffusion flux fields by the continuity condition (7). The velocity field should also comply with the prescribed velocity on $A_{V}$. An admissible lattice diffusion flux field is divergence-free in the grain interior; an admissible grain boundary flux field is such that condition (6) is satisfied at the grain boundary junctions. The proof that the functional (18) is stationary for the exact fields can be found in (McMeeking and Kuhn, 1992) and (Cocks, 1996), and elements of the proof are provided in Appendix A.1.

The kinematic minimum principle is analogous to the Potential Energy Theorem for elastic solids (Duvaut, 1990; Doghri, 2000). In the context of thermodynamic extremal principles (Fischer et al., 2014), the kinematic variational principle corresponds to the Minimum Principle for the the Dissipation Potential", and we refer to (Fischer et al., 2014; Hackl and Fischer, 2008) for a comparison of this principle to other thermodynamic extremal principles.

We consider the situation where the macroscopic strain rate $\dot{\boldsymbol{E}}$ is prescribed via velocity boundary conditions on the external surface of the aggregate. In this case $A=A_{V}$ and $A_{T}=\emptyset$, and thus $I=\Psi$. Evaluation of the dissipation potential for the exact fields together with Hill's condition (13) leads to the equality (see Eq. (72) in Appendix A.1):

$$
\Psi=\frac{1}{2} \boldsymbol{S}: \dot{\boldsymbol{E}}=G \dot{\boldsymbol{E}}: \dot{\boldsymbol{E}}
$$

Let $\Psi^{c}$ be the value of the dissipation potential calculated for admissible, not-necessarily exact fields. By analogy with Eq. (20), we define the corresponding estimate of the shear viscosity, $G^{+}$, as:

$$
\Psi^{c}=G^{+} \dot{\boldsymbol{E}}: \dot{\boldsymbol{E}} .
$$

According to the minimum principle, $\Psi \leq \Psi^{c}$, and therefore $G^{+}$constitutes an upper bound on the shear viscosity: $G^{+} \geq G$. 


\subsection{Statical variational principle}

Consider again an aggregate subject to prescribed velocity boundary conditions on the portion $A_{V}$ of its external surface, and to prescribed traction boundary conditions on the portion $A_{T}$ of its external surface. The complementary statical variational principle states that, among all admissible fields of stress and diffusion potential, the actual ones minimise the functional:

$$
J=\Phi-\frac{1}{V} \int_{A_{V}} \boldsymbol{T} \cdot \overline{\boldsymbol{v}} d A,
$$

where the dual dissipation potential $\Phi$ is given by:

$$
\Phi=\frac{1}{V}\left[\int_{V} \frac{1}{2} \frac{D_{l}}{\Omega k T} \nabla \mu \cdot \nabla \mu d V+\int_{A_{b}}\left(\frac{1}{2} \frac{\delta D_{b}}{\Omega k T} \nabla_{s} \mu \cdot \nabla_{s} \mu+\frac{1}{2 \eta} \sigma_{t}^{2}\right) d A\right] .
$$

In the second term of Eq. (22), $\overline{\boldsymbol{v}}$ represents the prescribed velocity on $A_{V}$. An admissible stress field satisfies the conditions of mechanical equilibrium in the grain interior and across grain boundaries. It also satisfies the traction boundary conditions on $A_{T}$. An admissible field of diffusion potential is such that $\mu=-\Omega \sigma_{n}$ on the grain boundary. The proof that the functional (22) is minimised for the exact fields is outlined in Appendix A.1. The statical variational principle is analogous to the Principle of Maximum Complementary Energy in elasticity (Doghri, 2000).

In the case where the macroscopic stress $\boldsymbol{S}$ is prescribed via traction boundary conditions on the external surface, $A_{T}=A, A_{V}=\emptyset$, and therefore $J=\Phi$. Evaluating the dual dissipation potential for the exact fields and using Hill's condition (13) leads to (see Eq. (74) of Appendix A.1):

$$
\Phi=\frac{1}{2} \boldsymbol{S}^{\prime}: \dot{\boldsymbol{E}}=\frac{\boldsymbol{S}^{\prime}: \boldsymbol{S}^{\prime}}{4 G} .
$$

Let $\Phi^{*}$ be the value of the dual dissipation potential calculated for admissible, notnecessarily exact fields. By analogy with Eq. (24), the corresponding estimate $G^{-}$of the shear modulus is defined as:

$$
\Phi^{*}=\frac{\boldsymbol{S}^{\prime}: \boldsymbol{S}^{\prime}}{4 G^{-}} .
$$

Since $\Phi \leq \Phi^{*}$, it follows that the shear viscosity $G^{-}$calculated from $\Phi^{*}$ constitutes a lower bound on the actual shear viscosity $G$ : $G^{-} \leq G$.

In Appendix A.2, it is shown that the exact shear modulus of an aggregate subject to affine velocity boundary conditions is larger or equal to the exact shear modulus of the same aggregate subject to uniform traction boundary conditions. This result is analogous to the corresponding result for the elastic modulus of random aggregates, see e.g. Huet (1990). As the number of grains in the random aggregate increases, the discrepancy between the moduli obtained with the two types of boundary conditions decreases as the effective behaviour becomes less sensitive of the detail fo the applied boundary conditions. By definition, the effective behaviour of a true RVE of the microstructure should be independent of the type of boundary conditions.

\section{Model for isotropic polycrystal}

We consider a randomly isotropic polycrystal with equiaxed grains and we assume that grain boundaries are randomly distributed and on average oriented perpendicular to the line joining the centres of gravity of two adjacent grains. Consequently, we consider 


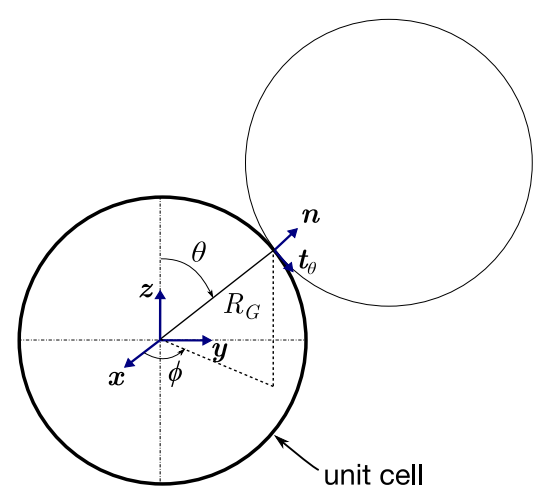

Figure 2: Unit cell containing one representative spherical grain. Grain boundaries are randomly distributed on the unit cell surface and oriented perpendicular to the line joining the centres of two adjacent cells.

as representative unit cell a spherical grain with radius $R_{G}$ and volume equal to the average grain volume which is in contact with an identical spherical grain at each point of its surface (McMeeking and Kuhn, 1992). The spherical grain model is convenient to illustrate the variational principles because it is amenable to simple analytical treatment. It also allows us to establish connections with classical results for Nabarro-Herring and Coble creep (Herring, 1950; Coble, 1963), which also relied on the spherical grain geometry, as well as more recent results by Onaka et al. (2001) who also accounted for non-zero friction coefficient. We will show that these classical results can be recovered as special case using the kinematic variational principle. In addition, new lower bounds based on the statical variational principle will be derived.

The spherical unit cell is represented in Fig. 2. The Cartesian components of the unit vector $\boldsymbol{n}$ normal to the representative sphere write

$$
\boldsymbol{n}=\left(\begin{array}{c}
\sin \theta \cos \phi \\
\sin \theta \sin \phi \\
\cos \theta
\end{array}\right)
$$

whereas the components of a unit vector $\boldsymbol{t}_{\theta}$ and $\boldsymbol{t}_{\phi}$ tangent to the grain boundary in the longitudinal and azimuthal directions write

$$
\boldsymbol{t}_{\theta}=\left(\begin{array}{c}
\cos \theta \cos \phi \\
\cos \theta \sin \phi \\
-\sin \theta
\end{array}\right), \quad \boldsymbol{t}_{\phi}=\left(\begin{array}{c}
-\sin \phi \\
\cos \phi \\
0
\end{array}\right)
$$

\subsection{Kinematic bound}

We first consider the case where the macroscopic strain rate is prescribed, and aim at estimating the corresponding macroscopic shear viscosity using the kinematic variational principle (Section 3.1). The macroscopic strain rate is chosen of the following form:

$$
\dot{\boldsymbol{E}}=\left(\begin{array}{ccc}
-\frac{\dot{E}}{2} & 0 & 0 \\
0 & -\frac{\dot{E}}{2} & 0 \\
0 & 0 & \dot{E}
\end{array}\right)
$$


and

$$
\begin{aligned}
\Psi= & \Psi_{l}+\Psi_{b}+\Psi_{s l} \\
= & \frac{1}{V_{G}} \int_{V_{G}} \frac{k T}{2 \Omega D_{l}} \boldsymbol{j}_{l} \cdot \boldsymbol{j}_{l} d V+\frac{1}{2 V_{G}} \int_{A_{G}} \frac{k T}{2 \Omega \delta D_{b}} \boldsymbol{j}_{b} \cdot \boldsymbol{j}_{b} d A \\
& \quad+\frac{1}{2 V_{G}} \int_{A_{G}} \frac{1}{2} \eta\left(\Delta v_{t}\right)^{2} d A,
\end{aligned}
$$

which should be calculated for a set of admissible velocity and diffusion fields compatible with the macroscopic strain rate. Compared to the general expression (19), the additional $(1 / 2)$ pre-factor in front of the the surface integrals accounts for the fact that contact points on the unit cell surface are shared between two grains.

A simple trial velocity field is obtained by assuming that the relative motion of the centres of adjacent grains comply with the macroscopic strain rate (Fleck et al., 1992; McMeeking and Kuhn, 1992):

$$
\Delta v_{i}=2 R_{G} \dot{E}_{i j} n_{j}
$$

This choice indeed satisfies equality (10), taking $A_{b}$ as the area of the spherical unit cell and accounting for the fact that contacts points are shared between two grains. (Note that in the spherical grain model, external boundaries of the aggregate are not represented and Eq. (9) cannot be used.) The normal and tangential components of $\Delta \boldsymbol{v}$ are calculated as

$$
\Delta v_{n}=\Delta \boldsymbol{v} \cdot \boldsymbol{n}=\frac{3}{2} R_{G} \dot{E}\left(\cos 2 \theta+\frac{1}{3}\right)
$$

and

$$
\Delta v_{t}=\Delta \boldsymbol{v} \cdot \boldsymbol{t}_{\theta}=-\frac{3}{2} R_{G} \dot{E} \sin 2 \theta
$$

Admissible diffusion fluxes in the lattice and on the grain boundary surface must be proposed in such a way that the continuity condition (7) is satisfied. For the spherical unit cell model, this condition particularises as:

$$
\Delta v_{n}=2 j_{l r}-\nabla \cdot \boldsymbol{j}_{b},
$$

since two identical grains meet at each contact point on the unit cell surface. We consider a class of trial diffusion fluxes of the following form:

$$
\begin{aligned}
& j_{l r}(r, \theta)=\frac{3}{4} \alpha r \dot{E}\left(\cos 2 \theta+\frac{1}{3}\right) \\
& j_{l \theta}(r, \theta)=-\frac{3}{4} \alpha r \dot{E} \sin 2 \theta \\
& j_{l \phi}(r, \theta)=0
\end{aligned}
$$

$$
j_{b \theta}(\theta)=\frac{1}{2} \beta R_{G}^{2} \dot{E} \sin (2 \theta), \quad j_{b \phi}(\theta)=0
$$

where $\alpha$ and $\beta$ are constant parameters. It can be verified that the trial lattice flux (35)-(37) is divergence-free and satisfies the symmetry condition $\boldsymbol{j}_{l}(0, \theta)=\mathbf{0}$. On the 
other hand, the trial grain-boundary flux (38) satisfies the symmetry condition $\boldsymbol{j}_{b}(0)=$ $\boldsymbol{j}_{b}(\pi / 2)=\mathbf{0}$. These fluxes satisfy the continuity condition (34) provided that the parameters $\alpha$ and $\beta$ are related by:

$$
\alpha-\beta=1 .
$$

Each pair $(\alpha, \beta)$ satisfying the above constraint thus leads to admissible diffusion flux fields compatible with the prescribed velocity discontinuity.

Inserting expressions (35)-(38) into expression (30), the diffusion contribution to the potential $\Psi$ is calculated:

$$
\Psi_{d}=\Psi_{l}+\Psi_{b}=\frac{1}{5} \frac{k T R_{G}^{3}}{\Omega \delta D_{b}} \dot{E}^{2}\left(\frac{3}{4 \Delta} \alpha^{2}+\beta^{2}\right),
$$

where we introduced the notation $\Delta=D_{l} R_{G} / \delta D_{b}$. The diffusion contribution to the upper bound on the viscosity is in turn calculated from Eq. (29):

$$
G_{d}^{+}=\frac{2 \Psi_{d}}{3 \dot{E}^{2}}=\frac{1}{15} \frac{k T R_{G}^{3}}{\Omega \delta D_{b}}\left(\frac{3}{2 \Delta} \alpha^{2}+\beta^{2}\right) .
$$

Grain boundary sliding bring an additional contribution to the potential (30), which, using Eq. (33) expresses as:

$$
\Psi_{s l}=\frac{9}{10} \dot{E}^{2} R_{G} \eta
$$

According to Eq. (29), the contribution of grain boundary sliding to the upper bound of viscosity is thus:

$$
G_{s l}^{+}=\frac{2 \Psi_{s l}}{3 \dot{E}^{2}}=\frac{3}{5} \eta R_{G},
$$

which is independent of the choice of parameters $\alpha$ and $\beta$.

The optimal (i.e. lowest) upper bound on the shear viscosity is obtained by finding the parameters $\alpha$ and $\beta$ which minimise the potential (40) under the constraint (39). This is easily achieved using a Lagrange multiplier, and leads to:

$$
\alpha=\left(1+\frac{3}{2 \Delta}\right)^{-1}, \beta=-\frac{3 \alpha}{2 \Delta}=-\left(1+\frac{2 \Delta}{3}\right)^{-1} .
$$

The diffusion contribution to the upper bound on the shear viscosity is illustrated in Fig. 3 as a function of parameter $\alpha$, for different value of $\Delta$. The optimal value of $\alpha$ (indicated by a circle on the figure) shifts from $\alpha=0$ for $\Delta \rightarrow 0$ (grain boundary diffusion-dominated, $\beta=-1$ ) to $\alpha=1$ for $\Delta \rightarrow \infty$ (lattice diffusion-dominated, $\beta=0$ ).

Using the optimal choice of parameters (44) in Eq. (41) and adding the sliding contribution (43), the lowest upper bound on the shear viscosity is finally obtained:

$$
G^{+}=G_{d}^{+}+G_{s l}^{+}=\frac{1}{15}\left(1+\frac{2 \Delta}{3}\right)^{-1} \frac{k T R_{G}^{3}}{\Omega \delta D_{b}}+\frac{3}{5} \eta R_{G} .
$$

When $\Delta \gg 1$, grain boundary diffusion is negligible relative to lattice diffusion, and the viscosity reduces to:

$$
G_{l}^{+}=\frac{k T R_{G}^{2}}{\Omega D_{l}}\left(\frac{1}{10}+\frac{3}{5} \eta_{l}\right)
$$

328 with $\eta_{l}=\eta\left(\frac{\Omega D_{l}}{k T R_{G}}\right)$. The first term on the right-hand side of Eq. (46) (lattice diffusion contribution) is identical to the result of Onaka et al. (2001), who used the same kinematic 


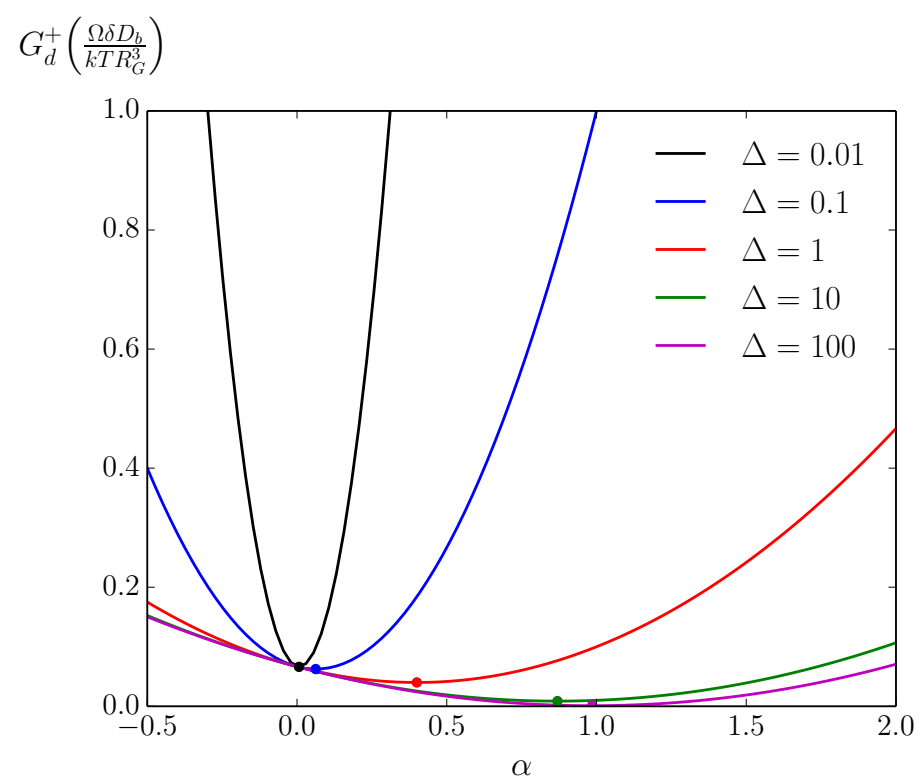

Figure 3: Diffusion contribution to the upper bound on the shear viscosity as a function of parameter $\alpha$, for different values of the ratio $\Delta=D_{l} R_{G} / \delta D_{b}$. The optimal value of $\alpha$, leading to the lowest upper bound, is indicated by a circle for each value of $\Delta$.

boundary conditions at the grain boundary and based their analysis on the concept of Somigliana's dislocations. Compared to these authors, our result for $G_{s l}^{+}$is however larger by a factor of two. The reason is that Onaka et al. did not consider the factor $1 / 2$ in front of the surface integral in Eq. (30). If grain boundary friction is neglected $(\eta=0)$, the result (46) also recovers Herring's result:

$$
G_{\text {Herring }}=\frac{1}{10} \frac{k T R_{G}^{2}}{\Omega D_{l}},
$$

(cf. Eq. (11) of (Herring, 1950)). Herring considered the problem of a spherical grain subject to affine velocity boundary conditions and represented the stress using spherical harmonics.

When $\Delta \ll 1$, lattice diffusion is negligible relative to grain boundary diffusion and the viscosity reduces to:

$$
G_{b}^{+}=\frac{k T R_{G}^{3}}{\Omega \delta D_{b}}\left(\frac{1}{15}+\frac{3}{5} \eta_{b}\right)
$$

with $\eta_{b}=\eta\left(\frac{\Omega \delta D_{b}}{k T R_{G}^{2}}\right)=\eta_{l} / \Delta$. With respect to the result of Onaka et al. (2001), our result for $G_{b}^{+}$is larger by a factor of two, for the same reason as mentioned below Eq. (46). If we further neglect grain boundary friction $(\eta=0)$, our result is close to the results of Coble who, via some approximations, obtained (cf. Eqs (13) and (14) of (Coble, 1963)):

$$
G_{\text {Coble }}=\frac{8}{3} \frac{\pi}{148} \frac{k T}{\Omega \delta D_{b}} R_{G}^{3}=\frac{1}{17.7} \frac{k T}{\Omega \delta D_{b}} R_{G}^{3} .
$$

In Appendix B.1 we show that the trial fields (35)-(38) with optimal parameters (44) constitute an exact solution to the boundary-value problem of a single spherical grain subject to prescribed interface velocity discontinuity of the form (32)-(33), in the sense 
that it is possible to calculate corresponding fields of diffusion potential and stress that satisfy all the constitutive and equilibrium equations in the grain. It is also verified in Appendix B.1 that the macroscopic deviatoric stress calculated from the shear viscosity (45) as $\boldsymbol{S}^{\prime}=2 G^{+} \dot{\boldsymbol{E}}$ coincides with the macroscopic deviatoric stress calculated by integrating the surface tractions according to Eq. (11).

\subsection{Statical bound}

We next consider the case of a prescribed deviatoric macroscopic stress of the form:

$$
\boldsymbol{S}=\left(\begin{array}{ccc}
-\frac{S}{2} & 0 & 0 \\
0 & -\frac{S}{2} & 0 \\
0 & 0 & S
\end{array}\right)
$$

and seek to find lower bounds on the shear viscosity using the statical variational principle (Section 3.2). Lower bounds on the shear viscosity are obtained from the potential $\Phi$ given by Eq. (23), which for the spherical unit cell is calculated as:

$$
\begin{gathered}
\Phi=\frac{1}{V_{G}} \int_{V} \frac{1}{2} \frac{D_{l}}{\Omega k T} \nabla \mu \cdot \nabla \mu d V+\frac{1}{2 V_{G}} \int_{A_{G}} \frac{1}{2} \frac{\delta D_{b}}{\Omega k T} \nabla_{s} \mu \cdot \nabla_{s} \mu d A \\
+\frac{1}{2 V_{G}} \int_{A_{G}} \frac{1}{2 \eta} \sigma_{t}^{2} d A,
\end{gathered}
$$

where $\mu$ and $\sigma_{t}$ are admissible diffusion potential and tangential stress fields in equilibrium with the macroscopic stress. According to Eq. (25), the lower bound on the shear modulus for the macroscopic loading (50) is given by:

$$
G^{-}=\frac{3 S^{2}}{8 \Phi}
$$

We consider a uniform trial stress within the grain, so that the traction field on the grain surface is simply given by:

$$
T_{i}=S_{i j} n_{j}
$$

Normal and tangent components of $\boldsymbol{T}$ are calculated as:

$$
T_{n}=\boldsymbol{T} \cdot \boldsymbol{n}=\sigma_{n}=\frac{3 S}{4}\left(\cos 2 \theta+\frac{1}{3}\right)
$$

and

$$
T_{t}=\boldsymbol{T} \cdot \boldsymbol{t}_{\theta}=\sigma_{t}=-\frac{3}{4} S \sin 2 \theta .
$$

The normal traction on the boundary in turn sets the trial field of diffusion potential on the boundary: $\mu=-\Omega \sigma_{n}=-\Omega T_{n}$. The choice (54)-(55) thus fully specifies the contributions of grain boundary diffusion and sliding to the potential $\Phi$ in Eq. (51). On the other hand, an admissible trial diffusion potential field within the bulk should ensure continuity of the diffusion potential, i.e. it should be such that: $\mu\left(R_{G}, \theta\right)=-\frac{3}{4} \Omega S\left(\cos 2 \theta+\frac{1}{3}\right)$ and $\frac{\partial \mu}{\partial \theta}\left(R_{G}, \theta\right)=\frac{3}{2} \Omega S \sin 2 \theta$. A parametrised set of trial fields satisfying these two conditions is given by:

$$
\mu(r, \theta)=\frac{3}{2} \frac{\Omega S}{R_{G}^{2}} r^{2}\left(-\frac{1}{2} \cos 2 \theta+\gamma\right)-\frac{3}{2} \Omega S\left(\gamma+\frac{1}{6}\right)
$$


where $\gamma$ is an adjustable parameter. In the bulk, the gradient of the trial field of diffusion potential (56) is calculated as:

$$
\begin{aligned}
\nabla_{r} \mu & =3 \frac{\Omega S r}{R_{G}^{2}}\left(-\frac{1}{2} \cos 2 \theta+\gamma\right) \\
\nabla_{\theta} \mu & =\frac{3}{2} \frac{\Omega S r}{R_{G}^{2}} \sin 2 \theta .
\end{aligned}
$$

The functional $\Phi$ in Eq. (51) is then calculated as:

$$
\Phi=\frac{\Omega \delta D_{b}}{k T R_{G}^{3}} S^{2}\left(\frac{9}{10}+\frac{27}{40} \Delta\left(1+4 \gamma^{2}+\frac{4 \gamma}{3}\right)+\frac{9}{40} \frac{1}{\eta_{b}}\right) .
$$

Lower bounds on the shear viscosity then follow from Eq. (52):

$$
G^{-}=\frac{k T R_{G}^{3}}{\Omega \delta D_{b}}\left(\frac{12}{5}+\frac{9}{5} \Delta\left(1+4 \gamma^{2}+\frac{4 \gamma}{3}\right)+\frac{3}{5} \frac{1}{\eta_{b}}\right)^{-1}
$$

The optimal (i.e. highest) lower bound is obtained by finding the value of the parameter $\gamma$ that minimises the potential $\Phi$ given by Eq. (59). This leads to

$$
\gamma=-\frac{1}{6}
$$

The evolution of the lower bound for $G(60)$ as a function of the parameter $\gamma$ is illustrated in Fig. 4, for $\eta_{l}=0.2$. The optimal value of $\gamma$, leading to the highest lower bound, is indicated by a circle on the figure.

For the optimal choice (61), the potential reduces to:

$$
\Phi=\frac{\Omega \delta D_{b}}{k T} \frac{S^{2}}{R_{G}^{3}}\left(\frac{2}{5}+\frac{4}{15} \Delta+\frac{1}{10} \frac{1}{\eta_{b}}\right),
$$

and the corresponding optimal lower bound is given by:

$$
G^{-}=\frac{k T R_{G}^{3}}{\Omega \delta D_{b}}\left(\frac{12}{5}+\frac{8}{5} \Delta+\frac{3}{5} \frac{1}{\eta_{b}}\right)^{-1} .
$$

In the limit where grain boundary diffusion is negligible relative to lattice diffusion, $\Delta \ll 1$ and shear viscosity is dominated by lattice diffusion and grain boundary sliding:

$$
G_{l}^{-}=\frac{k T R_{G}^{2}}{\Omega D_{l}}\left(\frac{8}{5}+\frac{3}{5 \eta_{l}}\right)^{-1} .
$$

In the limit where lattice diffusion is negligible relative to grain boundary diffusion, $\Delta \gg 1$ and shear viscosity is dominated by grain boundary diffusion and sliding:

$$
G_{b}^{-}=\frac{k T R_{G}^{3}}{\Omega \delta D_{b}}\left(\frac{12}{5}+\frac{3}{5 \eta_{b}}\right)^{-1} .
$$

In Appendix B.2, we show that the trial field of diffusion potential corresponding to the optimal value (61) of the parameter $\gamma$ is an exact solution to the boundary-value problem of a single spherical grain subject to prescribed tractions of the form (53), in the sense that it is possible to calculate lattice and grain-boundary diffusion fluxes that satisfy the constitutive relations and such that the lattice diffusion flux is divergence-free in the grain interior. 


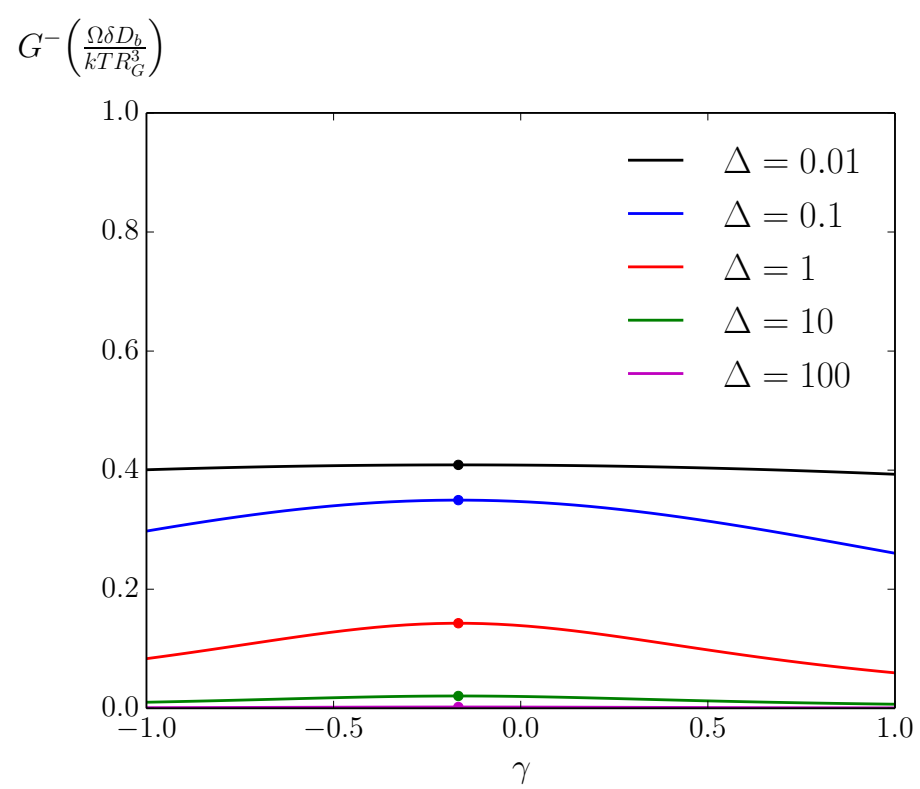

Figure 4: Lower bound on the shear viscosity as a function of parameter $\gamma$, for $\eta_{l}=0.2$ and different values of the ratio $\Delta=D_{l} R_{G} / \delta D_{b}$. The optimal value of $\gamma$, leading to the highest lower bound, in indicated by a circle for each value of $\Delta$.

Table 1: Effective shear viscosity $G$ in the lattice diffusion-dominated $\left(D_{l} R_{G} \gg \delta D_{b}\right)$ and grain boundary diffusion-dominated $\left(D_{l} R_{G} \ll \delta D_{b}\right)$ regimes, as predicted by different models.

\begin{tabular}{lcc}
\hline & $D_{l} R_{G} \gg \delta D_{b}$ & $D_{l} R_{G} \ll \delta D_{b}$ \\
\hline Upper bound & $\frac{1}{10} \frac{k T R_{G}^{2}}{\Omega D_{l}}+\frac{3}{5} \eta R_{G}$ & $\frac{1}{15} \frac{k T R_{G}^{3}}{\Omega \delta D_{b}}+\frac{3}{5} \eta R_{G}$ \\
Lower bound & $\left(\frac{8}{5} \frac{\Omega D_{l}}{k T R_{G}^{2}}+\frac{3}{5 \eta R_{G}}\right)^{-1}$ & $\left(\frac{12}{5} \frac{\Omega \delta D_{b}}{k T R^{3}}+\frac{3}{5 \eta R_{G}}\right)^{-1}$ \\
Herring (1951) & $\frac{1}{10} \frac{k T R_{G}^{2}}{\Omega D_{l}}$ & \\
Coble (1963) & & $\frac{1}{17.7} \frac{k T R_{G}^{3}}{\Omega \delta D_{b}}$ \\
\hline
\end{tabular}

\section{Discussion}

In the previous section, we used kinematic and statical variational principles to identify optimal upper and lower bounds on the shear viscosity of random aggregates represented by the spherical unit cell model. These optimal bounds were further shown to correspond to exact solutions of the boundary-value problem of a single spherical grain subject respectively to affine velocity jumps or uniform tractions on the grain boundary surface. Because of the spherical grain shape and simplified representation of grain-to-grain interactions through point contacts randomly distributed on the sphere surface, compatibility of velocity jumps and equilibrium of surface tractions across grain boundaries are also automatically fulfilled. In contrast, in a realistic random geometry of faceted grains, stresses corresponding to affine trial velocity jumps would not be equilibrated. Conversely, velocity and diffusion flux fields corresponding to uniform stress trial fields would not satisfy compatibility of deformation and conservation of mass. This is analogous to Voigt and Reuss bounds on the elastic modulus of heterogeneous media.

As illustrated below, the modulus corresponding to the spherical grain subject to affine velocity jump, Eq. (45) is larger or equal to the modulus corresponding the the spherical 
grain subject to uniform traction boundary condition, Eq. (63). This is consistent with the result established in Appendix A.2. The existence of two distinct values of the effective shear viscosity indicates that the spherical unit cell model does not constitute a RVE of the aggregate. Indeed, by definition, the effective behaviour of a RVE should be independent of whether macroscopic stress or macroscopic strain is prescribed. Mechanical analysis on a proper RVE is needed to assess whether a mean estimate between these two results would constitute an accurate estimate of the actual response of random aggregates of faceted grains.

A summary of our results in the two limiting regimes (lattice diffusion-dominated and grain boundary diffusion-dominated regimes) is presented in Table 1. Figure 5 shows normalised optimal upper and lower bounds on the shear viscosity drawn from Eqs (46) and (64) (lattice diffusion-dominated, Fig. 5a) or Eqs (48) and (65) (grain boundary diffusion-dominated, Fig. 5b) as a function of the dimensionless friction parameters $\eta_{l}$ or $\eta_{b}$. Note in particular that $\eta_{l}$ or $\eta_{b}$ increase when grain size decrease. Also note the different normalisation used for both the friction coefficient and the viscosity in terms of powers of $R_{G} \cdot \eta_{l}$ or $\eta_{b}$ can be interpreted as the ratio of a characteristic time for grain boundary sliding $\tau_{s l}=\frac{\eta \Omega R_{G}}{k T}$ (i.e. the timescale for relaxation of tangential stresses under an applied strain in the absence of diffusion) to a characteristic time for diffusion in the lattice, $\tau_{l}=\frac{R_{G}^{2}}{D_{l}}$, or along grain boundaries, $\tau_{b}=\frac{R_{G}}{\delta} \frac{R_{G}^{2}}{D_{b}}$.

As lattice diffusion dominates at large $R_{G}$, a decrease of $R_{G}$ brings about a transition from Fig. 5a to Fig. 5b around $R_{G}=\delta \frac{D_{b}}{D_{l}}$, for which $\tau_{l}=\tau_{b}$. This threshold also corresponds to $\eta_{l}=\eta_{b}$, which depends on the material properties. For example, if $\delta \frac{D_{b}}{D_{l}}=$ $10^{-6} \mathrm{~m}$, the threshold occurs at $R_{G}=1 \mu \mathrm{m}$, and if $\eta \frac{\Omega D_{l}}{k T}=10^{-7} \mathrm{~m}$, it corresponds to $\eta_{l}=\eta_{b}=0.1$ : the relevant parts of the curves are then the range $\eta_{l}<0.1$ in Fig. 5a and the range $\eta_{b}>0.1 \mathrm{in} \mathrm{Fig.} \mathrm{5b.} \mathrm{The} \mathrm{threshold} \mathrm{corresponding} \mathrm{to} R_{G}=1 \mu \mathrm{m}$ moves to the right if $\eta$ is larger. Reliable experimental data on the value to be postulated for the grain boundary friction coefficient $\eta$ in polycrystals are very scarce. As mentioned in the Introduction, arguments linked to the mechanisms of climb and glide of GBDs suggest that, for common grain sizes, $\eta_{l} \ll 1$ and $\eta_{b} \ll 1$. Nonetheless, $\eta_{l}$ and $\eta_{b}$ can become large in nanomaterials. As an example, from creep tests on $\mathrm{ZrO}_{2}$ at $1400^{\circ} \mathrm{C}$ under $3 \mathrm{MPa}$, Kim at al. (Kim et al., 2004) estimated $\eta_{b} \approx 0.5$ for $\mathrm{ZrO}_{2}$ when $R_{G}=0.35 \mu \mathrm{m}$ (on the basis of a two-dimensional model assuming a regular hexagonal lattice of grains). Hence, the range of scale chosen for the abscissae in Fig. 5 can be considered relevant for nanostructured materials.

For a fixed grain size, upper and lower bounds both monotonically increase with $\eta$. In Fig. 5a, the upper bound coincides with Herring's result for $\eta_{l}=0$ and increases linearly with $\eta_{l}$ with a slope of $3 / 5$. In Fig. 5 b, the upper bound is close to Coble's result for $\eta_{b}=0$ and increases with the same slope. This follows from the kinematic approach, according to which the tangential velocity is prescribed, and the contribution of grain boundary sliding to macroscopic stress scales linearly with the friction coefficient. In contrast, the lower bound is a non-linear function of the friction coefficient, with $G_{l}^{-}=G_{b}^{-}=0$ for $\eta_{l}=\eta_{b}=0$ and $G_{l}^{-}=\frac{5}{8} \frac{k T R_{G}^{2}}{\Omega D_{l}}$ or $G_{b}^{-}=\frac{5}{12} \frac{k T R_{G}^{3}}{\Omega \delta D_{l}}$ when $\eta_{l}$ or $\eta_{b} \rightarrow \infty$. These two limits respectively correspond to infinite and zero tangential velocity discontinuity at the grain boundary under prescribed tangential stress. The uncertainty on the mean estimate between the bounds is particularly large when $\eta_{l}$ or $\eta_{b}$ tend to zero (if $\eta \neq 0$, a domain of dominance of lattice diffusion always prevails below a certain threshold).

Interestingly, there is a particular value of the friction coefficient at which the curves 


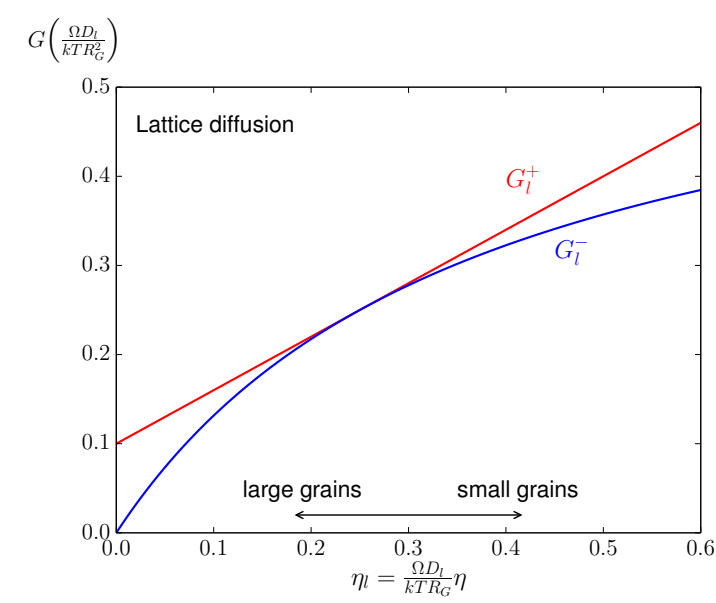

(a)

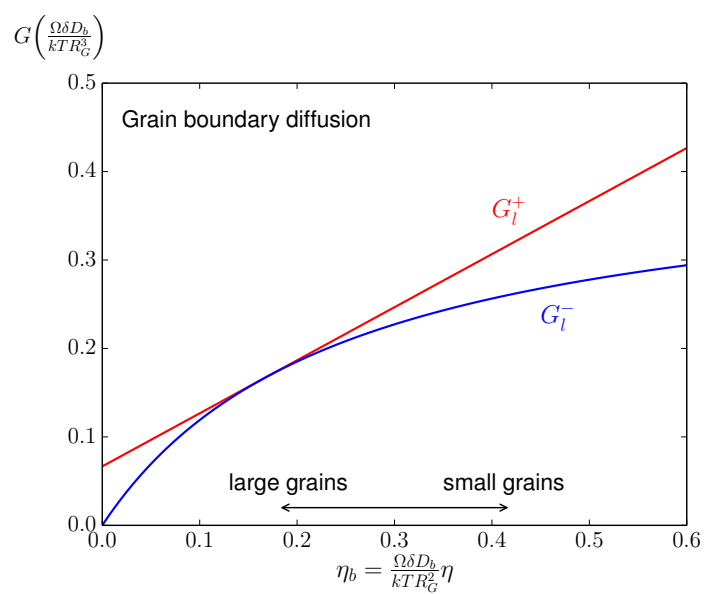

(b)

Figure 5: (a) Bounds on the shear viscosity drawn from Eqs (46) and (64). (b) Bounds on the shear viscosity drawn from Eqs (48) and (65).

for the two bounds are tangent to one another:

$$
G_{l}^{+}=G_{l}^{-}=\frac{1}{4} \frac{k T R_{G}^{2}}{\Omega D_{l}}=\frac{5}{2}\left(G_{l}^{+}\right)_{\eta_{l}=0}=\eta R_{G}
$$

when $\eta_{l}=\frac{1}{4}$, and

$$
G_{b}^{+}=G_{b}^{-}=\frac{1}{6} \frac{k T R_{G}^{3}}{\Omega \delta D_{b}}=\frac{5}{2}\left(G_{b}^{+}\right)_{\eta_{b}=0}=\eta R_{G}
$$

when $\eta_{b}=\frac{1}{6}$. The condition for the tangency of the curves is discussed in Appendix C. It is shown that there is a particular value of $\eta$ for which the traction field calculated when boundary velocity jumps are prescribed by macroscopic strain rate is identical to the traction field corresponding to prescribed uniform tractions, and vice versa. For that particular value of $\eta$, the affine relation (31) between velocity and macroscopic strain rate and the uniform traction condition (53) hold simultaneously. The velocity jump vectors and traction vectors are then parallel to each other, and the shear viscosity is $G=\eta R_{G}$, independent of the value of other material coefficients. The particular value (66) coincides with the "unrelaxed" solution obtained by Herring by simultaneously assuming uniform stress and strain rate within the grain (see Eq. (7) in (Herring, 1950)). For steady-state creep, our results show that this particular solution can be recovered for a particular value of the friction coefficient $\eta$.

We investigate the scaling of the shear viscosity with grain size by fitting Eqs (46), (64), (48) and (65) with a power law in terms of the grain size. Specifically, the dependence on $\eta_{l}$ or $\eta_{b}$ of the apparent grain size sensitivity exponent $p$ was calculated via the relationship

$$
p_{l}^{+}=\frac{d \ln G_{l}^{+}}{d \ln R_{G}}=\frac{d G_{l}^{+}}{d R_{G}} \frac{R_{G}}{G_{l}^{+}}
$$

and the like for the other bounds. The results are presented in Fig. 6. For zero grain boundary friction, $G_{l}^{+}$scales with the square of the grain size (Herring's result), whereas $G_{b}^{+}$scales with the third power of the grain size (Coble's result). In the limit of high $\eta_{l}$ or $\eta_{b}$, the upper bound is dominated by grain boundary sliding and scaling exponent $p$ tends 


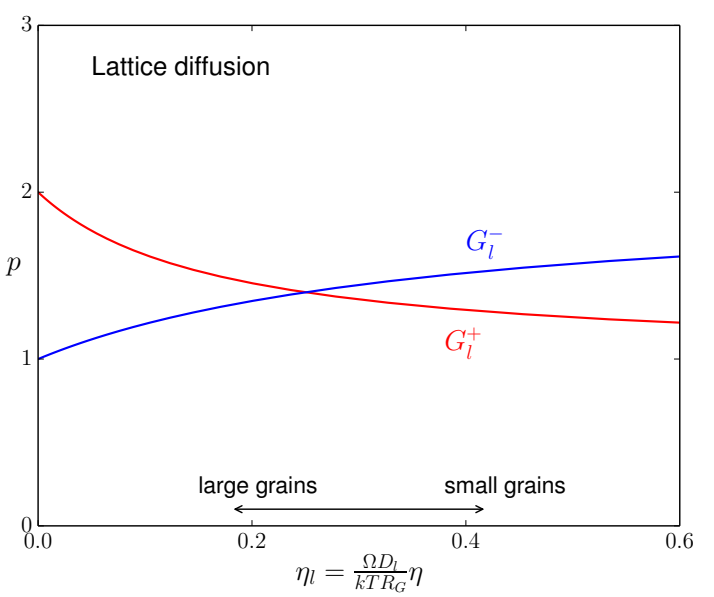

(a)

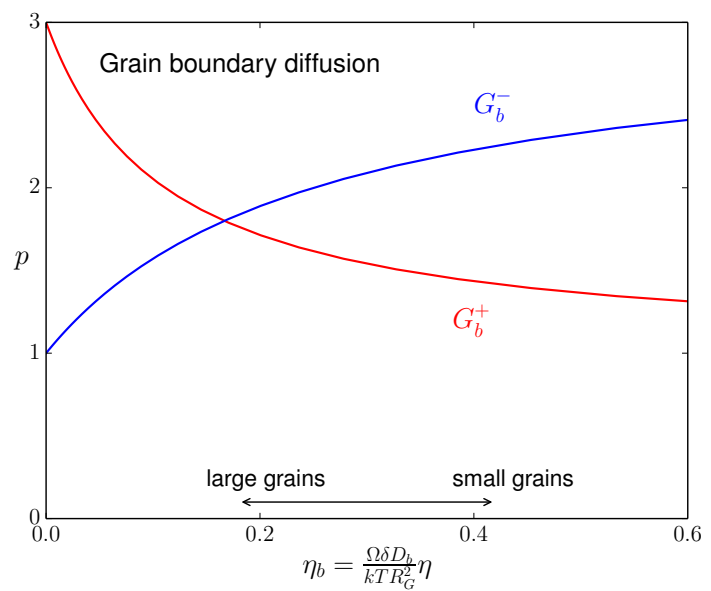

(b)

Figure 6: (a) Grain size sensitivity exponent of the bounds (46) and (64). (b) Grain size sensitivity exponent of the bounds (48) and (65).

to 1 . In the same limit, sliding becomes effectively inoperative in the lower bound and the scaling exponent tends to 2 or 3 . The mean between the two curves varies in the ranges $1.5 \geq p_{l} \geq 1.4$ and $2 \geq p_{b} \geq 1.8$. These curves cast doubt on the validity of the method of identification of the dominant creep mechanism based on the grain size dependence of creep rate: dominance of lattice diffusion if $p=2$; dominance of grain boundary diffusion if $p=3$; dominance of grain boundary friction forces if $p=1$. Experimentally, $p$ values close to 2 were much more frequently reported than $p$ values close to 3 (Coble, 1963; Cannon and Sherby, 1977; Cannon and Langdon, 1988; Mishra et al., 1988; Wang, 2000; Ruano et al., 2003).

In the literature, most experimental studies of creep under low stress reported creep rates significantly higher than the predictions of Herring's and Coble's models (Cannon and Sherby, 1977; Cannon and Langdon, 1988; Mishra et al., 1988; Wang, 2000). It may be tempting to link this discrepancy to the outcomes of the present work showing that classical models provide only an upper bound for shear viscosity and that the lower bound may be very low if the friction coefficient $\eta$ is small. Unfortunately, very little is known today about the value to be ascribed to $\eta$. In addition, in many cases, microstructural observations after creep testing or after superplastic straining showed evidence of the occurrence of Rachinger sliding (usually referred to as "grain boundary sliding (GBS)") (Wang, 2000; Chokshi, 2002; Ruano et al., 2003). Meaningful comparison with experimental creep data then requires a model involving the possibility of a strain contribution due to GBS. Forthcoming work will aim at addressing this issue as well as the effect of grain shape anisotropy and the difference between the present model and previous models based on periodic lattices of grains in two- or three-dimension (Gibbs, 1966; Raj and Ashby, 1971; Beere, 1976, 1978; Spingarn and Nix, 1978; Greenwood, 1985, 1992; Burton, 1994; Kim et al., 2003, 2004).

\section{Conclusion}

By revisiting classical models for NHC creep under the light of variational principles, the present work reveals that the widely acknowledged Herring's and Coble's predictions 
for viscosity dominated by lattice and/or grain boundary diffusion are actually upper bounds arising from the use of affine trial velocity discontinuity at interfaces, and that conversely lower bounds can be accessed via the use of uniform trial stresses in the grains. To the best of our knowledge these lower bounds have not been identified earlier in the literature. If the grain is represented by a sphere, the lowest upper bound and the highest lower bound are two independent exact solutions of the governing equations within the grain. The existence of such bounds challenges the validity of the classical method of identification of the dominant creep mechanism from the grain size sensitivity exponent. The work also emphasizes the role of grain boundary friction in diffusional viscosity.

\section{Appendix A. Variational formulations of the boundary-value problem}

\section{Appendix A.1. Principle of virtual powers}

Consider a polycrystalline aggregate occupying a volume $V$ with external surface $A$. The aggregate is subject to prescribed traction $\overline{\boldsymbol{T}}$ on $A_{T}$, and to prescribed velocity $\overline{\boldsymbol{v}}$ on $A_{V}$, with $A_{T} \cup A_{V}=A$ and $A_{T} \cap A_{V}=\emptyset$. Let $\sigma_{n}^{*}$ and $\sigma_{t}^{*}$ be normal and tangential interface stresses in equilibrium with traction $\boldsymbol{T}^{*}$ on the external surface. Let $\Delta v_{n}^{c}$ and $\Delta v_{t}^{c}$ be normal and tangential interface velocity jumps kinematically compatible with velocity $\boldsymbol{v}^{c}$ on the external surface. The Principle of Virtual Powers expresses as (Needleman and Rice, 1980; McMeeking and Kuhn, 1992; Cocks, 1996):

$$
\int_{A} \boldsymbol{T}^{*} \cdot \boldsymbol{v}^{c} d A=\int_{A_{b}}\left(\sigma_{n}^{*} \Delta v_{n}^{c}+\sigma_{t}^{*} \Delta v_{t}^{c}\right) d A .
$$

Using the divergence theorem together with relations (3), (4), (6) and (7), Eq. (69) can be rewritten as:

$$
\int_{A} \boldsymbol{T}^{*} \cdot \boldsymbol{v}^{c} d A=\int_{V}-\frac{\boldsymbol{j}_{l}^{c}}{\Omega} \cdot \nabla \mu^{*} d V+\int_{A_{b}}\left(-\frac{\boldsymbol{j}_{b}^{c}}{\Omega} \cdot \nabla_{s} \mu^{*}+\sigma_{t}^{*} \Delta v_{t}^{c}\right) d A
$$

In this expression, $\mu^{*}=-\Omega \sigma_{n}^{*}$ on $A_{b}$, and $\boldsymbol{j}_{l}^{c}$ and $\boldsymbol{j}_{b}^{c}$ are related to $\Delta v_{n}^{c}$ by Eq. (7) on $A_{b}$, with $\boldsymbol{\nabla} \cdot \boldsymbol{j}_{l}^{c}=0$ in the grain interior. We have also assumed that the net fluxes $\left(\boldsymbol{j}_{l}^{c} \cdot \boldsymbol{n}\right)$ and $\left(\boldsymbol{j}_{b}^{c} \cdot \boldsymbol{n}\right)$ vanish on the external surface.

Following Cocks (1996), let us identify the traction $\boldsymbol{T}^{*}$, stress $\sigma_{t}^{*}$ and diffusion potential $\mu^{*}$ in Eq. (70) with the exact fields, and identify the compatible fields of velocity $\boldsymbol{v}^{c}$, diffusion fluxes $\boldsymbol{j}_{l}^{c}$ and $\boldsymbol{j}_{b}^{c}$, and velocity jump $\Delta v_{t}^{c}$ with small perturbations $\delta \boldsymbol{v}, \delta \boldsymbol{j}_{l}, \delta \boldsymbol{j}_{b}$ and $\delta \Delta v_{t}$ about the exact solution. In particular, the perturbation $\delta \boldsymbol{v}$ vanishes on $A_{V}$. Then, relation (70) becomes:

$$
V \delta I \equiv \int_{V} \frac{k T}{\Omega D_{l}} \boldsymbol{j}_{l} \cdot \delta \boldsymbol{j}_{l} d V+\int_{A_{b}}\left(\frac{k T}{\Omega \delta D_{b}} \boldsymbol{j}_{b} \cdot \delta \boldsymbol{j}_{\boldsymbol{b}}+\eta \Delta v_{t} \delta \Delta v_{t}\right) d A-\int_{A_{T}} \overline{\boldsymbol{T}} \cdot \delta \boldsymbol{v}=0,
$$

where we have also used the constitutive relations (2), (5) and (8). This expression shows that the functional $I$ defined by Eq. (18) is stationary for the exact fields. It can also be shown that the stationary point corresponds to a minimum, see McMeeking and Kuhn (1992) for the proof. In the particular case where $A=A_{V}$ and $A_{T}=\emptyset$, evaluating the principle of virtual powers (70) for the exact fields also gives the following equality:

$$
\int_{A} \boldsymbol{T} \cdot \overline{\boldsymbol{v}} d A=\int_{V} \frac{k T}{\Omega D_{l}} \boldsymbol{j}_{l} \cdot \boldsymbol{j}_{l} d V+\int_{A_{b}}\left(\frac{k T}{\Omega \delta D_{b}} \boldsymbol{j}_{b} \cdot \boldsymbol{j}_{b}+\eta\left(\Delta v_{t}\right)^{2}\right) d A=2 V \Psi .
$$


Let us now identify the compatible velocity and diffusion fluxes in Eq. (70) with the exact fields, and identify the equilibrated fields of traction, stress and diffusion potential with small perturbations $\delta \boldsymbol{T}, \delta \mu$, and $\delta \sigma_{t}$ about the exact solution. In particular, the perturbation $\delta \boldsymbol{T}$ vanishes on $A_{T}$. Then, relation (70) yields:

$$
V \delta J \equiv \int_{V} \frac{D_{l}}{\Omega k T} \boldsymbol{\nabla} \mu \cdot \delta \boldsymbol{\nabla} \mu d V+\int_{A_{b}}\left(\frac{\delta D_{b}}{\Omega k T} \boldsymbol{\nabla} \mu_{s} \cdot \delta \boldsymbol{\nabla} \mu_{s}+\frac{\sigma_{t}}{\eta} \delta \sigma_{t}\right) d A-\int_{A_{V}} \overline{\boldsymbol{v}} \cdot \delta \boldsymbol{T}=0,
$$

where we have used the constitutive relations (2), (5) and (8). This expression shows that the functional $J$ defined by Eq. (22) is stationary for the exact fields. It can also be shown that the stationary point corresponds to a minimum. In the particular case where $A=A_{T}$ and $A_{V}=\emptyset$, evaluating the principle of virtual powers (70) for the exact fields gives the following equality:

$$
\int_{A} \boldsymbol{T} \cdot \overline{\boldsymbol{v}} d A=\int_{V} \frac{D_{l}}{\Omega k T} \boldsymbol{\nabla} \mu \cdot \boldsymbol{\nabla} \mu d V+\int_{A_{b}}\left(\frac{\delta D_{b}}{\Omega k T} \boldsymbol{\nabla}_{s} \mu \cdot \boldsymbol{\nabla}_{s} \mu+\frac{\sigma_{t}^{2}}{\eta}\right) d A=2 V \Phi .
$$

It can further be shown that $I+J=0$ for the exact fields. From Eqs (18) and (22), direct calculation gives:

$$
\begin{aligned}
V(I+J)= & \frac{1}{2} \int_{V} \frac{k T}{\Omega D_{l}} \boldsymbol{j}_{l} \cdot \boldsymbol{j}_{l} d V+\frac{1}{2} \int_{A_{b}}\left(\frac{k T}{\Omega \delta D_{b}} \boldsymbol{j}_{b} \cdot \boldsymbol{j}_{b}+\eta\left(\Delta v_{t}\right)^{2}\right) d A \\
& +\frac{1}{2} \int_{V} \frac{D_{l}}{\Omega k T} \boldsymbol{\nabla} \mu \cdot \boldsymbol{\nabla} \mu d V+\frac{1}{2} \int_{A_{b}}\left(\frac{\delta D_{b}}{\Omega k T} \boldsymbol{\nabla}_{s} \mu \cdot \nabla_{s} \mu+\frac{\sigma_{t}^{2}}{\eta}\right) d A \\
& -\int_{A_{F}} \overline{\boldsymbol{T}} \cdot \boldsymbol{v} d A-\int_{A_{V}} \boldsymbol{T} \cdot \overline{\boldsymbol{v}} d A .
\end{aligned}
$$

Using the constitutive relations (2), (5) and (8), the latter equation becomes:

$$
V(I+J)=\int_{V}-\frac{\boldsymbol{j}_{l}}{\Omega} \cdot \nabla \mu d V+\int_{A_{b}}\left(-\frac{\boldsymbol{j}_{b}}{\Omega} \cdot \nabla_{s} \mu+\sigma_{t} \Delta v_{t}\right) d A-\int_{A} \boldsymbol{T} \cdot \boldsymbol{v} d A,
$$

which vanishes by virtue of the principle of virtual powers (70) calculated for the exact fields. It also follows that $\Psi+\Phi=\frac{1}{V} \int_{A} \boldsymbol{T} \cdot \boldsymbol{v} d S=\boldsymbol{S}: \dot{\boldsymbol{E}}$, where the last equality follows from Hill's condition (13).

Appendix A.2. Comparison of the effective moduli for affine and uniform traction boundary conditions

We use the variational principles to show that the effective shear modulus for an aggregate subject to affine velocity boundary conditions is larger or equal to the modulus of the same aggregate subject to uniform traction boundary conditions. The proof follows Huet (1990), who demonstrated the analogous result in the context of elastic heterogeneous media.

Let us consider two distinct boundary-value problems for the same aggregate:

1. Problem 1: the aggregate is subject to affine boundary conditions of the form $\boldsymbol{v}_{1}=$ $\dot{\boldsymbol{E}}_{1} \cdot \boldsymbol{x}$ on $A$. Solution fields for this problem are written $\boldsymbol{v}_{1}, \boldsymbol{j}_{l 1}, \boldsymbol{j}_{b 1}, \boldsymbol{\sigma}_{1}$, and $\mu_{1}$.

2. Problem 2: the aggregate is subject to uniform traction boundary conditions of the form $\boldsymbol{T}=\boldsymbol{S}_{2} \cdot \boldsymbol{n}$ on $A$. Solution fields for this problem are written $\boldsymbol{v}_{2}, \boldsymbol{j}_{l 2}, \boldsymbol{j}_{b 2}, \boldsymbol{\sigma}_{2}$ and $\mu_{2}$. 
Using the fields $\boldsymbol{\sigma}_{2}$ and $\mu_{2}$ as admissible fields in the statical variational formulation of Problem 1 (section 3.2), we have the following inequality:

$$
-J_{1}\left[\boldsymbol{\sigma}_{2}, \mu_{2}\right] \leq-J_{1}\left[\boldsymbol{\sigma}_{1}, \mu_{1}\right]=I_{1}\left[\boldsymbol{v}_{1}, \boldsymbol{j}_{l 1}, \boldsymbol{j}_{b 1}\right],
$$

where we also used the result $I_{1}+J_{1}=0$ for the exact fields of Problem 1. From the definition (22) of the functional $J$, the left-hand side of Eq. (77) can be rewritten as:

$$
\begin{aligned}
-J_{1}\left[\boldsymbol{\sigma}_{2}, \mu_{2}\right]= & -\frac{1}{V}\left[\int_{V} \frac{1}{2} \frac{D_{l}}{\Omega k T} \boldsymbol{\nabla}_{2} \cdot \boldsymbol{\nabla} \mu_{2} d V\right. \\
& \left.+\int_{A_{b}}\left(\frac{1}{2} \frac{\delta D_{b}}{\Omega k T} \boldsymbol{\nabla}_{s} \mu_{2} \cdot \boldsymbol{\nabla}_{s} \mu_{2}+\frac{\sigma_{t 2}^{2}}{2 \eta}\right) d A-\int_{A}\left(\boldsymbol{S}_{2} \cdot \boldsymbol{n}\right) \cdot \boldsymbol{v}_{1} d A\right] \\
= & -\Phi_{2}\left[\boldsymbol{\sigma}_{2}, \mu_{2}\right]+\boldsymbol{S}_{2}: \dot{\boldsymbol{E}}_{1} \\
= & -\frac{\boldsymbol{S}_{2}: \boldsymbol{S}_{2}}{4 G_{2}}+\boldsymbol{S}_{2}: \dot{\boldsymbol{E}}_{1},
\end{aligned}
$$

where we also used Eq. (9) and (24). In the latter equation, $G_{2}$ is the effective shear modulus for Problem 2. On the other hand, the right-hand side of Eq. (78) can be expressed as:

$$
I_{1}\left[\boldsymbol{v}_{1}, \boldsymbol{j}_{l 1}, \boldsymbol{j}_{b 1}\right]=\Psi_{1}\left[\boldsymbol{v}_{1}, \boldsymbol{j}_{l 1}, \boldsymbol{j}_{b 1}\right]=G_{1} \dot{\boldsymbol{E}}_{1}: \dot{\boldsymbol{E}}_{1},
$$

with $G_{1}$ the effective shear modulus for Problem 1. Inserting Eqs (78) and (79) into Eq. (77) gives the inequality:

$$
-\frac{\boldsymbol{S}_{2}: \boldsymbol{S}_{2}}{4 G_{2}}+\boldsymbol{S}_{2}: \dot{\boldsymbol{E}}_{1} \leq G_{1} \dot{\boldsymbol{E}}_{1}: \dot{\boldsymbol{E}}_{1}
$$

For a fixed value of $\dot{\boldsymbol{E}}_{1}$, it is easily verified that the left-hand side of the above inequality reaches a maximum for $\boldsymbol{S}_{2}=2 G_{2} \dot{\boldsymbol{E}}_{1}$ (i.e. when the applied macroscopic stress for Problem 2 coincides with the macroscopic stress in Problem 1). For this particular value of $\boldsymbol{S}_{2}$, the inequality (80) becomes:

$$
G_{2} \dot{\boldsymbol{E}}_{1}: \dot{\boldsymbol{E}}_{1} \leq G_{1} \dot{\boldsymbol{E}}_{1}: \dot{\boldsymbol{E}}_{1}
$$

This shows that the shear modulus of an aggregate subject to uniform traction boundary conditions is lower or equal to the modulus of the same aggregate subject to affine velocity boundary conditions. The equality holds when the relationship between macroscopic stress and strain rate does not depend on the type of the boundary conditions, which is expected to occur when the aggregate is sufficiently large (i.e. contains a sufficiently large number of grains) and is statistically representative.

\section{Appendix B. Exact solutions of the boundary-value problem for a spherical grain}

Appendix B.1. Prescribed velocity jump at grain boundary

Using the optimal parameters (44), the diffusion flux field in the bulk lattice (35)-(36) becomes:

$$
\begin{aligned}
& j_{l r}(r, \theta)=\frac{3}{4}\left(1+\frac{3}{2 \Delta}\right)^{-1} r \dot{E}\left(\cos 2 \theta+\frac{1}{3}\right) \\
& j_{l \theta}(r, \theta)=-\frac{3}{4}\left(1+\frac{3}{2 \Delta}\right)^{-1} r \dot{E} \sin 2 \theta
\end{aligned}
$$


and the mean macroscopic stress is given by:

$$
S_{m}=-\frac{1}{12}\left(1+\frac{2 \Delta}{3}\right)^{-1} \frac{k T R_{G}^{3} \dot{E}}{\Omega \Delta D_{b}}+S_{0} .
$$


It can be verified that the deviatoric macroscopic stress $S_{i j}^{\prime}=S_{i j}-S_{m} \delta_{i j}$ obtained from Eqs (90)-(91) coincides with the macroscopic deviatoric stress calculated from the viscosity, $S_{i j}^{\prime}=2 G^{+} \dot{E}_{i j}$, with $G^{+}$given by Eq. (45), regardless of the choice of $S_{0}$.

One can always choose the constant $S_{0}$ such that the macroscopic mean stress vanishes and $\boldsymbol{S}=\boldsymbol{S}^{\prime}$ :

$$
S_{0}=\frac{1}{12}\left(1+\frac{2 \Delta}{3}\right)^{-1} \frac{k T R_{G}^{3} \dot{E}}{\Omega \Delta D_{b}} .
$$

For this choice of $S_{0}$, the interface stresses (88)-(89) particularise as:

$$
\begin{aligned}
\sigma_{n} & =\frac{1}{4} \frac{k T R_{G}^{3} \dot{E}}{\Omega \delta D_{b}}\left(1+\frac{2 \Delta}{3}\right)^{-1}\left(\cos 2 \theta+\frac{1}{3}\right), \\
\sigma_{t} & =-\frac{3}{2} \eta R_{G} \dot{E} \sin 2 \theta .
\end{aligned}
$$

Note that the choice of $S_{0}$ does not affect the tangential stress due to sliding, whose contribution to the macroscopic stress is always purely deviatoric.

\section{Appendix B.2. Prescribed tractions at grain boundary}

Using the optimal value (61) of parameter $\gamma$, the diffusion potential (56) becomes:

$$
\mu(r, \theta)=-\frac{3}{4} \frac{\Omega S}{R_{G}^{2}} r^{2}\left(\cos 2 \theta+\frac{1}{3}\right) .
$$

The optimal value (61) is the only one that ensures that $\nabla^{2} \mu=0$. The corresponding diffusion flux field in the bulk lattice is calculated from the constitutive relation (2):

$$
\begin{aligned}
& j_{l r}(r, \theta)=\frac{3}{2} \frac{D_{l}}{k T} \frac{\Omega S}{R_{G}^{2}} r\left(\cos 2 \theta+\frac{1}{3}\right) \\
& j_{l \theta}(r, \theta)=-\frac{3}{2} \frac{D_{l}}{k T} \frac{\Omega S}{R_{G}^{2}} r \sin 2 \theta,
\end{aligned}
$$

which is divergence-free. The surface flux along the grain boundary is obtained from relation (5):

$$
j_{b \theta}(\theta)=-\frac{3}{2} \frac{\delta D_{b}}{k T} \frac{\Omega S}{R_{G}} \sin 2 \theta .
$$

Normal and tangential velocity jumps are respectively calculated from the continuity condition (34) and the constitutive relation (8):

$$
\begin{aligned}
\Delta v_{n} & =\frac{9}{2} \frac{\delta D_{b}}{k T} \frac{\Omega S}{R_{G}^{2}}\left(1+\frac{2 \Delta}{3}\right)\left(\cos 2 \theta+\frac{1}{3}\right) \\
\Delta v_{t} & =-\frac{3}{4} \frac{S}{\eta} \sin 2 \theta .
\end{aligned}
$$

The traction field on the grain surface is prescribed by Eq. (53) and corresponds to a uniform stress in the grain. It follows from the above considerations that expression (96) for the diffusion potential is the exact solution of the spherical grain problem under prescribed traction at grain boundary. One can also verify that the macroscopic strain rate obtained by using the latter two equations into Eq. (10) is deviatoric and coincides with the macroscopic strain rate calculated directly from the lower bound, $\dot{E}_{i j}=\frac{S_{i j}}{2 G^{-}}$. 


\section{Appendix C. Point of tangency of upper and lower bound curves}

By comparing the stresses (94)-(95) under prescribed velocity conditions to the stresses (54)-(55) under prescribed tractions at grain boundary, we find that the two solutions are compatible (i.e., affine velocity jump at the interface and uniform stress within the grain occur simultaneously) only if the following condition is met:

$$
\eta=\frac{1}{6}\left(1+\frac{2 \Delta}{3}\right)^{-1} \frac{k T R_{G}^{2}}{\Omega \delta D_{b}} .
$$

Conversely, comparison of the velocity jumps (100)-(101) under prescribed tractions to the velocity jumps (32)-(33) under prescribed velocities leads to the exact same condition. It thus follows that the solutions under the two considered loading conditions coincide for the particular value (102) of $\eta$, and so does the effective shear viscosity.

Condition (102) can be particularised to the two limit cases:

- Lattice diffusion-dominated $(\Delta \rightarrow \infty)$ :

$$
\eta \frac{\Omega D_{l}}{k T R_{G}}=\eta_{l}=\frac{1}{4} .
$$

- Grain boundary diffusion-dominated $(\Delta \rightarrow \infty)$ :

$$
\eta \frac{\Omega \delta D_{b}}{k T R_{G}^{2}}=\eta_{b}=\frac{1}{6} .
$$

It can also be verified that, for the particular value (102) of $\eta$, the interface velocity vector and traction vector are parallel, i.e. $\frac{\Delta v_{n}}{T_{n}}=\frac{\Delta v_{t}}{T_{t}}=$ constant. Here, the constant is set by the friction coefficient itself, according to the model of grain boundary sliding (8):

$$
\boldsymbol{T}=\eta \Delta \boldsymbol{v} .
$$

Using relation (105) into expression (11) of the macroscopic stress, and accounting for expression (10) of the macroscopic strain rate, one directly obtains:

$$
S_{i j}=2 R_{G} \eta \dot{E}_{i j} .
$$

The same result is obtained by first inserting relation (105) into expression (10) of the macroscopic strain rate and then identifying the macroscopic stress using Eq. (11). The shear viscosity is thus given by $G=\eta R_{G}$, independent of the type of boundary condition and the value of other material properties. 


\section{References}

Arzt, E., Ashby, M., Verrall, R., 1983. Interface controlled diffusional creep. Acta Metallurgica 31, 1977-1989.

Ashby, M., 1969. On interface-reaction control of Nabarro-Herring creep and sintering. Scripta Metallurgica 3, 837-842.

Ashby, M., 1972. Boundary defects, and atomistic aspects of boundary sliding and diffusional creep. Surface Science 31, 498-542.

Ashby, M., Edward, G., Davenport, J., Verrall, R., 1978. Application of bound theorems for creeping solids and their application to large strain diffusional flow. Acta Metallurgica 26, 1379-1388.

Balluffi, R., Allen, S., Carter, W., 2005. Kinetics of Materials. Wiley.

Beere, W., 1976. Stress redistribution during Nabarro-Herring and superplastic creep. Metal Science 10, 133-139.

Beere, W., 1977. Grain-boundary sliding controlled creep: its relevance to grain rolling and superplasticity. Journal of Materials Science 12, 2093-2098.

Beere, W., 1978. Stresses and deformation at grain boundaries [and discussion]. Philosophical Transactions of the Royal Society of London A: Mathematical, Physical and Engineering Sciences 288, 177-196.

Bilde-Sørensen, J., Smith, D., 1994. Comment on "Refutation of the relationship between denuded zones and diffusional creep". Scripta Metallurgica et Materialia 30, 383-386.

Burton, B., 1994. Anisotropy of grain-boundary diffusional creep in a polycrystalline array with orthorhombic grain geometry. Philosophical Magazine A 69, 565-582.

Burton, B., 2002. Diffusional rotation of crystals about a common interface. Philosophical Magazine A 82, 51-64.

Cannon, W., 1972. The contribution of grain boundary sliding to axial strain during diffusion creep. Philosophical Magazine A 25, 1489-1497.

Cannon, W., Langdon, T., 1988. Creep of ceramics. Journal of Materials Science 23, 1-20.

Cannon, W., Sherby, O., 1977. Creep behavior and grain-boundary sliding in polycrystalline $\mathrm{Al}_{2} \mathrm{O}_{3}$. Journal of the American Ceramic Society 60, 44-47.

Chokshi, A., 2002. Diffusion creep in oxide ceramics. Journal of the European Ceramics Society 22, 2469-2478.

Coble, R., 1963. A model for boundary diffusion controlled creep in polycrystalline materials. Journal of Applied Physics 34, 1679-1682.

Cocks, A., 1994. The structure of constitutive laws for the sintering of fine grained materials. Acta Metallurgica et Materialia 42, 2191-2210. 
Cocks, A., 1996. Variational principles, numerical schemes and bounding theorems for deformation by Nabarro-Herring creep. Journal of the Mechanics and Physics of Solids 44, 1429-1452.

Cocks, A., Gill, S., Pan, J., 1998. Modelling microstructure evolution in engineering materials. Advances in Applied Mechanics 36, 81-162.

Crossman, F., Ashby, M., 1975. The non-uniform flow of polycrystals by grain-boundary sliding accommodated by power-law creep. Acta Metallurgica 23, 425-440.

Doghri, I., 2000. Mechanics of deformable solids. Linear and nonlinear, analytical and computational aspects. Springer.

Duvaut, G., 1990. Mécanique des milieux continus. Dunod.

Fischer, F., Svoboda, J., Petryk, H., 2014. Thermodynamic extremal principles for irreversible processes in materials science. Acta Materialia 67, 1-20.

Fleck, N., Kuhn, L., McMeeking, R., 1992. Yielding of metal powder bonded by isolated contacts. Journal of the Mechanics and Physics of Solids 40, 1139-1162.

Gibbs, G., 1966. Diffusion creep of a thin foil. The Philosophical Magazine: A Journal of Theoretical Experimental and Applied Physics 13, 589-593.

Gibbs, G., 1968. The role of grain-boundary sliding in high-temperature creep. Materials Science and Engineering 2, 269-272.

Gifkins, R., Langdon, T., 1970. Grain boundary displacements due to diffusional creep. Scripta Metallurgica 4, 563-566.

Gleiter, H., Hornbogen, E., Baro, G., 1968. The mechanism of grain boundary glide. Acta Metallurgica 16, 1053-1067.

Green, H., 1970. Diffusional flow in polycrystalline materials. Journal of Applied Physics 41, 3899-3902.

Greenwood, G., 1985. An analysis of the effect of multiaxial stresses and grain shape on Nabarro-Herring creep. Philosophical Magazine A 51, 537-542.

Greenwood, G., 1992. A formulation for anisotropy in diffusional creep. Proceedings of the Royal Society of London A 436, 187-196.

Hackl, K., Fischer, F., 2008. On the relation between the principle of maximum dissipation and inelastic evolution given by dissipation potentials. Proceedings of the Royal Society of London A 464, 117-132.

Herring, C., 1950. Diffusional viscosity of a polycrystalline solid. Journal of Applied Physics 21, 437-445.

Herring, C., 1971. Reply to "The driving force for diffusion". Scripta Metallurgica 5, $273-277$.

Hill, R., 1967. The essential structure of constitutive laws for metal composites and polycrystals. Journal of the Mechanics and Physics of Solids 15, 75-95. 
Hötzer, J., Seiz, M., Kellner, M., Rheinheimer, W., Nestler, B., 2019. Phase-field simulation of solid state sintering. Acta Materialia 164, 184-195.

Huet, C., 1990. Application of variational concepts to size effects in elastic heterogeneous bodies. Journal of the Mechanics and Physics of Solids 38, 813-841.

Keblinski, P., Wolf, D., Gleiter, H., 1998. Molecular-dynamics simulation of grainboundary diffusion creep. Interface Science 6, 205-212.

Kim, B.-N., Hiraga, K., Morita, K., Ahn, B.-W., 2004. Analysis of creep due to grainboundary diffusion in hexagonal microstructures. Philosophical Magazine 84, 32513262 .

Kim, B.-N., Hiraga, K., Morita, K., Chen, I.-W., 2005a. Rate of creep due to grainboundary diffusion in polycrystalline solids with grain-size distribution. Philosophical Magazine 85, 2281-2292.

Kim, B.-N., Hiraga, K., Morita, K., Yoshida, H., 2005b. Effect of viscous grain-boundary sliding on high-temperature deformation of nano-sized grains. Review on Advanced Materials Science 10, 54-58.

Kim, B.-N., Hiraga, K., Morita, K., Yoshida, H., Ahn, B.-W., 2009. Viscous grainboundary sliding with rotating particles or grains. Acta Materialia 57, 5730-5738.

Kim, B.-N., Morita, K., Hiraga, K., 2003. Rate of diffusion creep accompanied by grain boundary sliding in elongated microstructures. Materials Science and Engineering A $363,67-71$.

Lifshitz, I., 1963. On the theory of diffusion-viscous flow of polycrystalline bodies. Soviet Physics JETP 17, 909-920.

McMeeking, R., Kuhn, L., 1992. A diffusional creep law for powder compacts. Acta Metallurgica et Materialia 40, 961-969.

Mishra, R., Jones, H., Greenwood, G., 1988. Enhanced diffusional creep: The effect of grain growth. Scripta Metallurgica 22, 323-327.

Mori, T., Nakasone, Y., Taya, M., Wakashima, K., 1997. Steady-state creep rate of a composite: Two-dimensional analysis. Philosophical Magazine Letters 75, 359-365.

Mori, T., Onaka, S., Wakashima, K., 1998a. Role of grain-boundary sliding in diffusional creep of polycrystals. Journal of Applied Physics 83, 7547-7552.

Mori, T., Taya, M., Wakashima, K., 1998b. Steady-state creep of a composite analysed by an energy balance method. Philosophical Magazine Letters 78, 331-338.

Nabarro, F., 1948. Report of a conference on the strength of solids. The Physical Society, London 75.

Needleman, A., Rice, J., 1980. Plastic creep flow effects in the diffusive cavitation of grain boundaries. Acta Metallurgica 28, 1315-1332. 
Onaka, S., Huang, J., Wakashima, K., 1998. Kinetics of stress relaxation caused by the combination of interfacial sliding and diffusion: two-dimensional analysis. Acta Materialia $46,3821-3828$.

Onaka, S., Madgwick, A., Mori, T., 2001. Kinetics of diffusional creep discussed by energy dissipation and effect of grain-size distribution on the rate equations. Acta Materialia 49, 2161-2168.

Pan, J., Cocks, A., 1993. Computer simulation of superplastic deformation. Computational Materials Science 1, 95-109.

Rachinger, W., 1952-1953. Relative grain translations in the plastic flow of aluminium. J. Inst. Metals 81, 33.

Raj, R., Ashby, M., 1971. On grain boundary sliding and diffusional creep. Metallurgical Transactions 2, 1113-1127.

Ruano, O., Wadsworth, J., Sherby, O., 2003. Deformation of fine-grained alumina by grain boundary sliding accommodated by slip. Acta Materialia 51, 3617-3634.

Spingarn, J., Nix, W., 1978. Diffusional creep and diffusionally accommodated grain rearrangement. Acta Metallurgica 26, 1389-1398.

Suo, Z., 1997. Motions of microscopic surfaces in materials. Advances in Applied Mechanics 33, 193-294.

Wang, J., 2000. Investigation of the deformation mechanism in grain size-sensitive Newtonian creep. Acta Materialia 48, 1517-1531.

Wang, Y.-J., Ishii, A., Ogata, S., 2011. Transition of creep mechanism in nanocrystalline metals. Physical Review B 84, 224102.

Wei, Y., Bower, A., Gao, H., 2008. Recoverable creep deformation and transient local stress concentration due to heterogeneous grain-boundary diffusion and sliding in polycrystalline solids. Journal of the Mechanics and Physics of Solids 56, 1460-1483. 